\title{
DNAJC12-associated developmental delay, movement disorder, and mild hyperphenylalaninemia identified by whole-exome sequencing re-analysis
}

\author{
Danielle Veenma $^{1} \cdot$ Dawn Cordeiro $^{1} \cdot$ Neal Sondheimer $^{1,2,3} \cdot$ Saadet Mercimek-Andrews $\mathbb{D}^{1,2,3}$
}

Received: 20 March 2018 / Revised: 18 July 2018 / Accepted: 24 July 2018 / Published online: 23 August 2018

(c) European Society of Human Genetics 2018

\begin{abstract}
Hyperphenylalaninemia, movement disorder, and intellectual disability due to variants in DNAJC12 is a recently reported inherited neurotransmitter disorder. We report two new patients with this new genetic disorder. Patient 1 is a 6-year-11month-old boy with mild hyperphenylalaninemia and global developmental delay (GDD). Seventeen-year-old male sibling of patient 1 had GDD from the first year of life. He had mild hyperphenylalaninemia at 11.5 years of age following his younger brother's diagnosis. He had low levels of homovanillic acid and 5-hydroxyindolacetic acid in the cerebrospinal fluid. Whole-exome sequencing (WES) was normal in 2016. After the first description of DNAJC12-associated hyperphenylalaninemia, dystonia, and intellectual disability in 2017, WES re-analysis identified a homozygous c.58_59delGG (p.(Gly20Metfs*2)) variant in DNAJC12. His younger brother was homozygous for the same variant, confirming the diagnosis of DNAJC12-associated hyperphenylalaninemia, movement disorder, and intellectual disability. Mild hyperphenylalaninemia and GDD should warrant targeted DNAJC12 genetic testing for the early diagnosis of DNAJC12-associated hyperphenylalaninemia, movement disorder, and intellectual disability.
\end{abstract}

\section{Introduction}

Hyperphenylalaninemia, movement disorder, and intellectual disability due to variants in DNAJC12 (MIM\#606060) was reported for the first time in 2017 as a new autosomal recessively inherited neurotransmitter disorder (OMIM\#617384) [1]. Elevated phenylalanine and low cerebrospinal fluid (CSF) homovanillic acid (HVA) and 5hydroxyindolacetic acid (5-HIAA) levels are characteristic

Electronic supplementary material The online version of this article (https://doi.org/10.1038/s41431-018-0237-9) contains supplementary material, which is available to authorized users.

Saadet Mercimek-Andrews

saadet.andrews@sickkids.ca

1 Division of Clinical and Metabolic Genetics, The Hospital for Sick Children, 555 University Avenue, Toronto, ON M5G 1X8, Canada

2 Genetics and Genome Biology Program, Research Institute, The Hospital for Sick Children, 555 University Avenue, Toronto, ON M5G 1X8, Canada

3 Department of Pediatrics, University of Toronto, 555 University Avenue, Toronto, ON M5G 1X8, Canada biochemical features. Biallelic variants in DNAJC12 have been identified in all patients [1-4]. DNAJC12 functions as a co-chaperone to prevent the misfolding of proteins and interacts with phenylalanine, tyrosine, and neuronal tryptophan hydroxylases [1, 5]. The destabilization of these enzymes with subsequent loss of phenylalanine, tyrosine, and neuronal tryptophan hydroxylases activity leads to hyperphenylalaninemia and neurotransmitter deficiency $[1,5,6]$.

We report two siblings with GDD, cognitive dysfunction, hyperphenylalaninemia, movement disorder, and autism spectrum disorder (ASD). Both children had a homozygous variant in DNAJC12. Additionally, we reviewed the literature for all patients reported so far.

\section{Subjects and methods}

Written informed consent was obtained from the parents for both patients.

\section{Patient 1}

This 6-year-11-month-old boy was born at term after an uneventful pregnancy to consanguineous parents of 
Afghan descent. He came to medical attention following a positive newborn screening for phenylketonuria at 13 days of age (blood spot phenylalanine of $271 \mu \mathrm{mol} / \mathrm{L}$; cut-off <107). The confirmatory plasma phenylalanine was $249 \mu \mathrm{mol} / \mathrm{L}$ (reference 45-65). Urinary pterins and blood spot dihydropteridine reductase activity were normal. He was diagnosed with a mild hyperphenylalaninemia.

Phenylalanine levels were $<600 \mu \mathrm{mol} / \mathrm{L}$ throughout his follow-up. He was never placed on a phenylalaninerestricted diet, as our center only treats children whose phenylalanine levels exceed $600 \mu \mathrm{mol} / \mathrm{L}$. At 6 months of age, his parents reported concerns about his development. He sat independently and acquired a pincer grasp at 12 months of age. He walked independently at 3 years of age. He had 6 words at 3.5 years of age. He was diagnosed with autism spectrum disorder (ASD) at 2 years of age. He was prescribed sapropterin dihydrochloride (20 mg/kg per day) (a synthetic form of tetrahydrobiopterin, which is a cofactor for phenylalanine hydroxylase) at 6 years of age. Direct sequencing of $P A H$ was normal. Prolactin level was normal at 2.5 years of age $(12.7 \mu \mathrm{g} / \mathrm{L}$; reference 4.2-23).

\section{Patient 2}

This 17-year-old brother of Patient 1 was born at term after an uneventful pregnancy by emergency $\mathrm{C}$-section due to fetal distress and maternal fever. His newborn screening was reported to be normal by his parents. He had early infantile onset developmental delay. He walked at 18 months of age. He acquired his first words at 2.5 years of age. He had clinical diagnosis of attention deficit hyperactivity disorder at 4 years of age. He was diagnosed with ASD at 8 years of age. After his younger brother's diagnosis of mild hyperphenylalaninemia, he underwent plasma amino acid analysis revealing mild hyperphenylalaninemia $(538 \mu \mathrm{mol} / \mathrm{L}$; reference $45-65)$ at 11.5 years of age. CSF neurotransmitter analysis showed mildly decreased levels of HVA (119 nmol/L; reference 167-563) and 5-HIAA (10 nmol/L; reference 67-189). He was prescribed oral sapropterin dihydrochloride $(15 \mathrm{mg} / \mathrm{kg}$ per day) at 15 years of age. His parents reported subjective improvements in his behavior. Unfortunately, neuropsychological assessment prior to or on the sapropterin dihydrochloride therapy was not performed.

\section{Results}

Patient 2 underwent whole-exome sequencing (WES) at age 16 years in 2016. The test did not identify any underlying genetic condition. Following the first report of patients with DNAJC12 associated hyperphenylalaninemia, dystonia, and intellectual disability in 2017, patient 2's WES data were re-analyzed [1] and identified a homozygous 2 bp deletion, c.58_59delGG (p.(Gly20Metfs*2)), in DNAJC12 (NM_021800.2) (using HGVS description of sequence variants). His parents were heterozygous and his younger symptomatic brother was homozygous for this variant. This variant was included into the BioPKU database (http://www.biopku.org/pnddb/ search-results.asp) with the identification number PNDDB298, which is a publicly available database. These results confirmed the diagnosis of DNAJC12 associated GDD, cognitive dysfunction, hyperphenylalaninemia, and movement disorder in both brothers.

Both brothers were referred to the inherited neurotransmitter disease clinic for medical management. There was no history of autonomic dysfunction in both brothers. At school he performed at the level of a 3-4year-old child at 6 years and 9 months of chronological age. His weight and height were at the 60th percentile for age. His head circumference was at the 98th percentile. He had dystonia in his hands and feet. When he was 17 years old, his cognitive function was assessed to be at the level of a 4-5-year-old child. His weight was at the 60th, height at the 80th, and head circumference at the 90th percentiles for age. He had dystonia in his hands and feet and hyperkinetic movements. Both patients were prescribed L-dopa/carbidopa and 5hydroxytrypthopan therapy to a target dose of $10 \mathrm{mg} / \mathrm{kg}$ per day. Unfortunately, they did not reach target dose and this treatment was discontinued by his parents after 4 months. They continued taking oral sapropterin dihydrochloride.

\section{Discussion}

We report two new patients with DNAJC12 associated hyperphenylalaninemia, GDD, cognitive dysfunction, ASD, and dystonia. The patients were followed for more than 5 years for mild hyperphenylalaninemia and GDD. The onset of developmental concerns occurred in the first 6 months of life, which is the earliest age of onset reported so far. Patient 2 did not have positive newborn screening for phenylketonuria. The mild hyperphenylalaninemia in the older patient was only identified after the positive newborn screening in his younger brother. Despite infantile disease onset and having had no treatment for defects in neurotransmission, there was no loss of developmental milestones or cognitive functions nor progression in his dystonia nor Parkinsonian features for 17 years in the older patient.

There were 15 patients with DNAJC12 associated disease reported since 2017 [1-4]. Supplementary Tables 1, 2, 3 


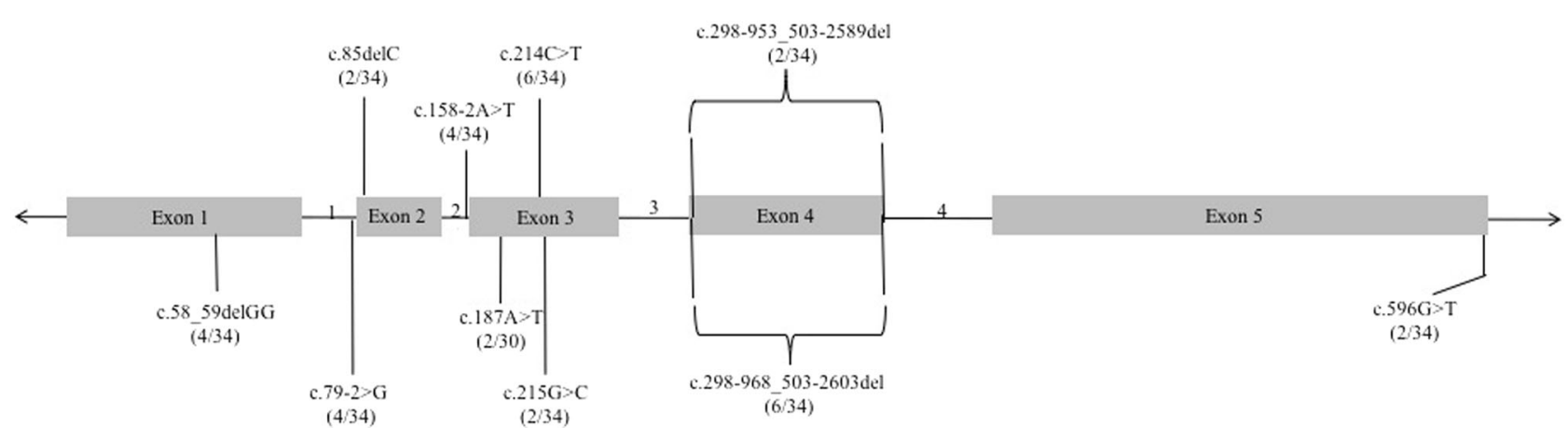

Fig. 1 Variants in DNAJC12. Schematic representation of all variants in DNAJC12 are depicted in Fig. 1 including our patients' variant. Allele frequency in the reported patients including our patients are given in parenthesis

summarized clinical features, biochemical and molecular genetic results, and outcome of all patients. Eleven additional patients were reported in a recent review article [7]. Ten patients had various degrees of developmental delay or cognitive dysfunction [1, 2, 4]. Four patients had dystonia, one patient had oculogyric crises, and four patients had Parkinsonism. All patients had mild hyperphenylalaninemia $[1,3,4,7]$. Newborn screening was normal in only two patients $[1,4]$. Our older patient's normal newborn screening for phenylketonuria suggests that plasma amino acid analysis should be performed in patients with GDD, behavioral problems, and/or movement disorder, since normal newborn screening is not sufficient to exclude DNAJC12 associated hyperphenylalaninemia, dystonia, and intellectual disability.

Ten patients underwent CSF 5-HIAA and CSF HVA measurements. Interestingly, CSF 5-HIAA was lower than CSF HVA levels in the majority of the patients [1-4]. Lower CSF 5-HIAA compared to CSF HVA levels could be associated with a stronger interaction between DNAJC12 and tryptophan hydroxylase. DNAJC12 interacts with an additional 44 proteins, 21 of which are localized in the mitochondria, suggesting that DNAJC12 might have an important role in the mitochondrial process [8]. Pyruvate carboxylase, a key regulator of tricarboxylic acid cycle, was the most commonly identified mitochondrial protein interacting with DNAJC12 [8]. A wider range of clinical and biochemical phenotypes may be associated with DNAJC12 variants in the future.

Eighty-nine percent of the DNAJC12 variants are truncating [1-4]. All variants in DNAJC12 are depicted in Fig. 1. All variants in DNAJC12, reported so far, are registered in the BioPKU database including our patients' variant (http://www.biopku.org/pnddb/search-results.asp). The Genome Aggregation Database (http://gnomad.broadinstitute. org/gene/ENSG00000108176) (accessed on 28 November 2017) identifies 20 truncating variants in DNAJC12 in a heterozygous state in 85 individuals [9]. Large number of individuals with mild hyperphenylalaninemia $(<600 \mu \mathrm{mol} / \mathrm{L}$ plasma phenylalanine levels without phenylalanine-restricted diet) are followed in our center. These two patients were the only mild hyperphenylalaninemia patients with GDD. These findings join a growing consensus that DNAJC12-associated hyperphenylalaninemia, dystonia, and intellectual disability might be a rare inherited neurotransmitter disorder.

Subjective improvements in cognitive and motor functions have been observed by parents after the initiation of sapropterin dihydrochloride monotherapy. Combined sapropterin dihydrochloride and L-dopa/carbidopa with or without 5-hydroxytryptophan therapy initiated in the first year of life resulted in normal neurodevelopment in three patients. Conversely, combined therapy initiated after the second year of life resulted in improvements in the cognitive and motor functions, but did not lead to normal neurodevelopmental outcome in three patients [1-3]. It is likely that early treatment is key to reversing or preventing neurodevelopmental disorders in patients with DNAJC12-associated hyperphenylalaninemia, dystonia, and intellectual disability.

In conclusion, we report two new patients with GDD, cognitive dysfunction, hyperphenylalaninemia, dystonia, and ASD caused by homozygous variant in DNAJC12. Mild hyperphenylalaninemia and GDD should warrant targeted DNAJC12 genetic testing for the early diagnosis and initiation of treatment to improve neurodevelopmental outcome.

Acknowledgements We thank the parents for allowing us to present their children's results.

\section{Compliance with ethical standards}

Conflict of interest The authors declare that they have no conflict of interest.

\section{References}

1. Anikster Y, Haack TB, Vilboux T, Pode-Shakked B, Thöny B, Shen $N$, et al. Biallelic mutations in DNAJC12 cause 
hyperphenylalaninemia, dystonia, and intellectual disability. Am J Hum Genet. 2017;100:257-66.

2. Straniero L, Guella I, Cilia R, Parkkinen L, Rimoldi V, Young A, et al. DNAJC12 and dopa-responsive nonprogressive parkinsonism. Ann Neurol. 2017;82:640-6.

3. van Spronsen FJ, Himmelreich N, Rüfenacht V, Shen N, Vliet DV, Al-Owain M, et al. Heterogeneous clinical spectrum of DNAJC12deficient hyperphenylalaninemia: from attention deficit to severe dystonia and intellectual disability. J Med Genet. 2017. https://doi. org/10.1136/jmedgenet-2017-104875.

4. de Sain-van der Velden MGM, Kuper WFE, Kuijper MA, van Kats LAT, Prinsen HCMT, Balemans ACJ, et al. Beneficial effect of $\mathrm{BH}_{4}$ treatment in a 15-year-old boy with biallelic mutations in DNAJC12. JIMD Rep. 2018. https://doi.org/10.1007/8904_2017_86.
5. Huttlin EL, Ting L, Bruckner RJ, Gebreab F, Gygi MP, Szpyt J, et al. The BioPlex Network: a systematic exploration of the human interactome. Cell. 2015;162:425-40.

6. Dekker SL, Kampinga HH, Bergink S. DNAJs: more than substrate delivery to HSPA. Front Mol Biosci. 2015;2:35.

7. Blau N, Martinez A, Hoffmann GF, Thöny B. DNAJC12 deficiency: a new strategy in the diagnosis of hyperphenylalaninemias. Mol Genet Metab. 2018;123:1-5.

8. Choi J, Djebbar S, Fournier A, Labrie C. The co-chaperone DNAJC12 binds to $\mathrm{Hsc70}$ and is upregulated by endoplasmic reticulum stress. Cell Stress Chaperon. 2014;19:439-46.

9. Lek M, Karczewski KJ, Minikel EV, Samocha KE, Banks E, Fennell T, et al. Analysis of protein-coding genetic variation in 60,706 humans. Nature. 2016;536:285-91. 